\title{
Stochastic Measures of Arbitrage
}

\author{
Alejandro Balbás \\ Departamento de Economía de la Empresa, Universidad Carlos III de Madrid \\ C/ Madrid 126, 28903 Getafe, Madrid, Spain \\ e-mail: balbas@emp.uc3m.es \\ María José Muñoz-Bouzo \\ Departamento de Matemáticas Fundamentales, UNED \\ $C /$ Senda del Rey s/n, 28040 Madrid, Spain \\ e-mail mjmunoz@mat.uned.es
}

\begin{abstract}
Empirical research has provided evidence supporting the existence of arbitrage opportunities in real financial markets although market imperfections are often the main reason to explain these empirical deviations. Consequently, recent literature has turned the attention to imperfect markets in order to extend the most significant results on asset pricing. This paper develops several stochastic measures providing relative arbitrage earnings available in a financial market. The measures allow us to take into account different type of frictions. They are introduced by means of several dual pairs of vector optimization problems. Primal problems permit us to characterize the arbitrage absence even in an imperfect market and they also provide optimal arbitrage portfolios if the arbitrage absence fails. Dual ones allow us to extend the risk-neutral valuation methodology for imperfect and noarbitrage free markets and provide new interpretations for the measures in terms of "frictions effect" or "committed errors" in the valuation process.
\end{abstract}

Key Words: Arbitrage, risk-neutral probability measure, market integration, vector optimization, dual problem, duality gap.

AMS subject classification: 90A09, 90B50, 90C29.

\section{Introduction}

Most of the theory of portfolio choice and asset pricing models under uncertainty is built under some ideal hypotheses on asset prices that are

We thank Monique Florenzano for her help and interest. We are also grateful to the unknown reviewers whose comments and suggestions have led to important improvements.

Partially funded by Comunidad Autónoma de Madrid (ref: CAM 07T/0027/2000) and Spanish Ministry of Science and Technology (ref: BEC2000-1388-C04)

Manuscript received: January 2002. Final version accepted: October 2002. 
not totally fulfilled in practice. Consequently, some of the empirical research presents discrepancies with respect to some theoretical results. So for instance, concerning the arbitrage absence, Protopapadakis and Stoll (1983) identify situations where the Law of One price is violated in dealing with spot and future prices, and Kamara and Miller (1995) tests of the put-call parity lead them to conclude that arbitrage opportunities exist and are available for some traders. Focusing on market integration, Lee and Nayar (1993), Chen and Knez (1995) and Kempf and Korn (1998) show the existence of some kind of segmentation when the absence of cross-market arbitrage is being empirically tested.

Market imperfections (bid-ask spread, other transaction costs, illiquidity that makes a continuous trading rather difficult, etc.) are often (but not always) the main reason to explain these empirical deviations, and previous literature has already focused on them. For instance, Toft (1996) analyzes how to replicate a call option in a model with transaction costs.

Concerning the arbitrage absence in the imperfect market case, Jouini and Kallal (1995) paper is a very interesting contribution. The authors consider sublinear pricing rules instead of linear ones, and extend the classical results on risk-neutral valuation and the martingale property for models without free lunches. Another important papers related to pricing methods in imperfect markets are due to Chateauneuf et al. (1996) and De Wagenaere and Wakker (2001), where sublinear pricing rules are considered once more, and they are represented by means of Choquet integrals.

The present paper focuses on two main objectives. First, we will provide some stochastic measures of the degree of violation of the arbitrage absence that allow us to incorporate several alternative hypotheses on market frictions. Therefore, the measures characterize the absence of arbitrage or yield concrete arbitrage strategies even in the imperfect market case. Later, we will develop a risk-neutral valuation methodology that also applies for imperfect markets and, furthermore, generates proxies of risk-neutral probability measures in no arbitrage free models. The approach provides, not only the existence of risk-neutral probability measures, but also a procedure to compute them.

A purpose of this paper is to define measures in a dynamic setting. Attention is directed to measure in a discrete (finite or infinite) time model. We start with the idea that there is an arbitrage opportunity in the model if there exist two consecutive dates such that there is an arbitrage opportunity 
in this period, in the line of Balbás et al. (2002b).

Another purpose of this paper is to measure arbitrage opportunities in monetary terms, following the approach of Balbás and Muñoz-Bouzo (1998). Although there are very interesting treatments of the arbitrage measurement in a static framework (see for instance Chen and Knez (1995), Kempf and Korn (1998) or Balbás and Muñoz-Bouzo (1998)) and all of them might be extended for dynamic models, the Balbás and Muñoz-Bouzo approach seems to be flexible enough when dealing with empirical tests or applications. This is pointed out in Balbás et al. (1999) and Balbás et al. (2000). The first paper shows the interest of an arbitrage measurement when pricing in markets whose illiquidity makes it difficult to apply standard procedures, while the second draws on the Balbás and Muñoz-Bouzo measure to empirically analyze the level of integration among several markets.

Measuring the arbitrage in monetary terms seems to reflect another advantages. So, Balbás et al. (2002a) shows that traders' objectives may be usually incorporated to the measure, and Jaschke (1998) illustrates how the sequential arbitrage measurement in bonds markets may be an useful tool when computing proxies for the term structure of interest rates.

We will look for relative arbitrage profits. We proceed in two ways. The first assumes the absence of short sale restrictions. The central idea of the stochastic measure $l$ is to test in each period the minimum initial investment over the price of the exchanged assets, needed to purchase a portfolio that generates nonnegative payoff in almost every state of nature.

The second way takes place in the presence of short sale restrictions. We first introduce for each period an infinite dimensional linear optimization problem. A solution to this problem yields to the maximum expected arbitrage profit obtained by an agent in presence of short sale restrictions in all the assets, i.e, the agent cannot sell what he/she does not have. We then consider the maximum of the found maxima among all the portfolios of the bounds of short position with price one at the first date of the period. This leads to a measure $\mathfrak{M}$ which depends only on the period.

The results show that some introduced optimization problems reflect very interesting properties. An optimal arbitrage portfolio $x$ leading to the maximum expected relative profit $\mathfrak{M}$ also leads to a stochastic measure $m$. This measure can be interpreted as the maximum arbitrage profit relative to the price of the sold assets in almost every state of nature. To show this, 
we prove that $x$ solves a vector optimization problem in which the objective function is non differentiable and takes values in an infinite-dimensional $L^{2}$ space. Finally, we prove that $x$ also solves the multiobjective optimization problem introduced to define $l$. This allows us to relate $l, m$ and $\mathfrak{M}$.

The measures $m$ and $\mathfrak{M}$ can be also obtained from a dual approach, useful for several reasons. First, it provides a proxy for discount factors (or equivalent concepts, like the risk neutral probabilities or the state prices) in no-arbitrage free economies, and thus, we extend the methodology of risk-neutral valuation and can compute "right prices" for the assets and the errors committed by the agents. Second, in a context of imperfect markets, the arbitrage may be only apparent but not real, and a theory that provides discount factors under not ideal assumptions makes the models more flexible and realistic. Finally and besides, we obtain some results on the absence of duality gaps for some vector optimization problems that do not hold for general vector problems (see for instance Khanh (1995) or Balbás and Guerra (1996)). This might be interesting in Optimization Theory.

This article is organized as follows. Section 2 introduces the optimization programs leading to define the measures $l$ and $\mathfrak{M}$. In Section 3 we prove the solvability of the programs associated to the measure $\mathfrak{M}$ and define the stochastic measure $m$. Section 4 provides some dual optimization problems such that their solutions lead to the measures $m$ and $\mathfrak{M}$. This allows us to relate $m$ and $\mathfrak{M}$ with the stochastic discount factors, and therefore, with some dynamic results of the existing literature. We provide other interpretations of the measure $m$ in Section 5 . We also state the solvability of the optimization problem leading to the measure $l$ and relate both measures $m$ and $l$. The concluding section contains miscellaneous remarks and extensions of the main results.

\section{Measurement of the arbitrage opportunities}

Consider a market for trading $n$ securities at a countable number of times $0,1, \ldots$ As usual, there is some finite or infinite set $\Omega$ of states of the world. For each date $t$, a $\sigma$-algebra $\Sigma_{t}$ of subsets of $\Omega$ denotes the set of events corresponding to the information available at time $t$. We adopt the usual convention that $\Sigma_{t} \subseteq \Sigma_{s}$ whenever $t \leq s$. Finally, $P$ is a probability measure defined on a $\sigma$-algebra of subsets of $\Omega$ containing all the $\sigma$-algebras $\Sigma_{t}$. A strategy trading at time $t$ is $x=\left(x_{1}, x_{2}, \ldots, x_{n}\right) \in\left(L^{2}\left(\Sigma_{t}\right)\right)^{n}$. This 
restriction of attention to square integrable random variables is made for expositional and mathematical ease. Let $p^{t}=\left(p_{1}^{t}, p_{2}^{t}, \ldots, p_{n}^{t}\right) \in\left(L^{2}\left(\Sigma_{t}\right)\right)^{n}$ the prices vector at time $t$. Then, for a strategy trading at time $t$, the value at date $t$ is the sum $\sum_{i=1}^{n} x_{i} p_{i}^{t}$ and the value at date $t+1$ is the sum $\sum_{i=1}^{n} x_{i} p_{i}^{t+1}$.

Definition 2.1. The portfolio $x \in\left(L^{2}\left(\Sigma_{t}\right)\right)^{n}$ is said to be an arbitrage opportunity (second type) at date $t$ if there exists $A \in \Sigma_{t}$ with $P(A)>0$ such that $\sum_{i=1}^{n} x_{i} p_{i}^{t+1} \geq 0$ a.e. in $A$ and $\sum_{i=1}^{n} x_{i} p_{i}^{t}<0$ a.e. in $A$.

If $x \in\left(L^{2}\left(\Sigma_{t}\right)\right)^{n}$ is an arbitrage opportunity at date $t$, it allows an agent to increase with positive probability consumption at date $t$, and increase (or at least not decrease) consumption at date $t+1$. The latter definition extends the notion of arbitrage opportunity of the second type (Ingersoll (1987)).We will extend the analysis in the concluding section in order to incorporate the opportunities of the first type which can be introduced as the portfolios $x \in\left(L^{2}\left(\Sigma_{t}\right)\right)^{n}$, with nonnegative price at time $t+1$ that are positive with positive probability, and with zero price at time $t$.

In the existing literature, the notion of absence of arbitrage in the different models varies from some authors to others. Under very natural assumptions and for a finite number of dates, it may be proved that the absence of arbitrage of both types at every date is equivalent to the absence of simple free lunches in the sense of Harrison and Kreps (1979). However, for an infinite number of trading dates the absence of free lunches is a stronger property (see Clark (1993) for a general treatment on this point). From an economic viewpoint the absence of arbitrage is much more intuitive than the absence of free lunch, but from a theoretical point of view the second concept may be characterized in very general frameworks by the existence of equivalent martingale measures. Anyway, in this paper we turn our attention to measure relative profits from arbitrage strategies of the second type because we retrieve the economic interpretation, and recent literature has shown that arbitrage free models may be also characterized by a martingale approach (see Balbás et al. (2002)). Thus we adopt the following definition.

Definition 2.2. We say that the model is arbitrage free if there are no arbitrage opportunities at every date.

We are interested in defining two stochastic processes to analyze attainable relative arbitrage profits in each date and in almost every state of 
nature. For expositional reasons, a brief synopsis of these approaches to measure arbitrage opportunities follows. Most technical details have been left to sections below, so all definitions stated in this synopsis are subject to results to be proved.

From now on, fix the period $t, t+1$. Let us introduce the following assumptions:

A.1 For every $i=1, \ldots, n, p_{i}^{t} \in L^{2}\left(\Sigma_{t}\right)$ and there exists $k_{i} \in \mathbb{R}$ such that $p_{i}^{t}(\omega)>k_{i}>0$

A.2 For every $i=1, \ldots, n, p_{i}^{t+1} \in L_{+}^{\infty}\left(\Sigma_{t+1}\right)$, and there exists $k \in \mathbb{R}$ such that $p_{1}^{t+1}(\omega)>k>0$.

Previous assumptions will be relaxed in future sections (see for instance Section 6). The assumptions imposed to $p_{1}$ are verified if we assume that the first security is a riskless asset. As Harrison and Kreps (1979) show, this is not a very restrictive assumption. In fact, assuming that one of the securities always has strictly positive price, we can use the price of this security as the numeraire (see Section 6).

FIRST MEASURE.

Consider for every $x \in\left(L^{2}\left(\Sigma_{t}\right)\right)^{n}$ the function,

$$
g(x, \omega)=\left\{\begin{array}{lll}
\frac{-\sum_{i=1}^{n} x_{i}(\omega) p_{i}^{t}(\omega)}{\sum_{i=1}^{n}\left|x_{i}(\omega)\right| p_{i}^{t}(\omega)} & \text { if } \quad x(\omega) \neq 0 \\
0 & \text { if } x(\omega)=0
\end{array}\right.
$$

If $x$ is an arbitrage opportunity at date $t$ and $A \in \Sigma_{t}$ is as in Definition 1, then $g(x, \omega)$ is the quotient whenever $\omega \in A$ between the profit generated by $x$ and the price of all exchanged assets, both computed at date $t$.

Since $|g(x, \omega)| \leq 1$ holds for every $\omega \in \Omega$ and every portfolio $x$, and $g(x, \quad)$ is $\Sigma_{t}$-measurable, it follows that $g(x, \quad) \in L^{2}\left(\Sigma_{t}\right)$ for every $x \in$ $\left(L^{2}\left(\Sigma_{t}\right)\right)^{n}$. Consider the following non-differentiable vector optimization 
problem:

$$
\max g(x, \quad) \quad \text { s.t } \quad \sum_{i=1}^{n} x_{i} p_{i}^{t+1} \geq 0
$$

Problem $(I)$ describes the process of identifying the portfolio which maximizes in almost every state of nature the arbitrage profit at date $t$ in relation to the price of all interchanged assets.

Definition 2.3. Assume that Problem $(I)$ is solvable. We define the first measure of the level of arbitrage opportunities by

$$
l^{t}(\omega)=g(x, \omega)
$$

where $x \in\left(L^{2}\left(\Sigma_{t}\right)\right)^{n}$ is an optimal solution in $(I)$.

It is important to point out that $l^{t}$ gives an information useful to analyze the absence of arbitrage in markets with transaction costs. Therefore, we have an alternative to the approach of Prisman (1986). In fact, if we assume that transaction costs are a.e. determined by the price $V(\omega)=\sum_{i=1}^{n}\left|x_{i}(\omega)\right| p_{i}^{t}(\omega)$ of the exchanged assets, then, once $(I)$ has been solved, we can subtract costs from the maximum profit an investor can obtain. Furthermore, it will be shown that market imperfections proposed by Jouini and Kallal (1995) can be incorporated in our model too (see Section 6).

We must prove the consistency of the above definition or, equivalently, that Problem $(I)$ is solvable. From a point of view of Optimization Theory, Problem $(I)$ presents some difficulties. Notice that the objective function is non linear and non differentiable. Moreover, it is an $L^{2}\left(\Sigma_{t}\right)$-valued function and we are looking for a strong maximum, i.e., an upper bound for the usual partial order in $L^{2}\left(\Sigma_{t}\right)$. In sharp contrast to the vast body of literature on efficiency in multiobjective optimization, see for instance Khanh (1995), little is known about strong optimality. There are some conditions for the existence of strong optima in vector optimization problems, see for instance Zowe (1975), but they usually do not apply in practical situations. In much of the cases, an strong optimum does not necessarily exist. However, we prove in Section 5 that there exists an optimal strong solution of Problem (I).

\section{SECOND MEASURE.}

We now assume that we are in presence of short sale restrictions. We 
introduce a couple of combined optimization programs to analyze arbitrage profits. The first one is a pair of primal-dual programs for each short sale restriction $h=\left(h_{1}, \ldots h_{n}\right) \in\left(L_{+}^{2}\left(\Sigma_{t}\right)\right)^{n}$ :

$$
\begin{aligned}
\max -\sum_{i=1}^{n} & \int_{\Omega} x_{i} p_{i}^{t} d P \\
\text { s.t. } & \sum_{i=1}^{n} x_{i} p_{i}^{t+1} \geq 0 \\
& x_{i} \geq-h_{i} \quad i=1, \ldots, n \\
& x_{i} \in L^{2}\left(\Sigma_{t}\right) \quad i=1, \ldots, n
\end{aligned}
$$

and its dual problem,

$$
\begin{aligned}
\min & \sum_{i=1}^{n} \int_{\Omega} h_{i} l_{i} d P \\
& \text { s.t. } E\left(f p_{i}^{t+1} \mid \Sigma_{t}\right)+l_{i}=p_{i}^{t} \\
& \quad f \in L_{+}^{2}\left(\Sigma_{t+1}\right), l_{i} \in L_{+}^{2}\left(\Sigma_{t}\right) \text { for every } i=1, \ldots, n
\end{aligned}
$$

where $f$ and $l_{i}, i=1, \ldots, n$ are decision variables and $E\left(f p_{i}^{t+1} \mid \Sigma_{t}\right)$ denotes the conditional expectation of $f p_{i}^{t+1}$ relative to $\Sigma_{t}$.

The problem $\left(I I_{h}\right)$ describes the process of identifying the portfolio (constrained by the bounds in a short position $h_{i} \geq 0$ ) which minimizes the initial expected investment needed to purchase a portfolio that generates a nonnegative payoff in almost every state of nature. Thus, a non zero optimal value in $\left(I I_{h}\right)$, provided its existence, represents the maximum expected profit obtained by an agent implementing arbitrage in such a way that he/she cannot sell more than $h_{i}$ units in each asset $i$.

Problems $\left(I I_{h}\right)$ and $\left(I I I_{h}\right)$ avoid some difficulties encountered when dealing with problem $(I)$ since their objective functions are linear and real valued. However, the variables take values in an $L^{2}$-space, with an empty interior of the positive cone unless $\Sigma_{t}$ is a finite $\sigma$-algebra. So, most of the usual conditions do not apply to establish the absence of duality gap. In Section 4 we establish conditions on $\Sigma_{t}$ under which there is no duality gap. These conditions are fulfilled in practical situations. When this absence of duality gap could not be stated, some considerations in Section 3 prove that 
we can consider an equivalent formulation in which the primal constraint space is an $L^{\infty}$-space; this is the only one of the $L^{p}$-spaces to have a positive cone with interior points (in its norm topology). In this case the dual variable space must be the dual Banach space for $L^{\infty}$, and thus will not be a function space. Nevertheless, the absence of duality gap can be always stated. This is the reason for considering in Section 6 such a topological framework for the pair dual $\left(I I_{h}\right)$ and $\left(I I I_{h}\right)$.

We denote by $\varphi(h)$ the optimum value in $\left(I I_{h}\right)$. We try to define a measure as the maximum attainable expected profit from an arbitrage opportunity among all the possible investors holding portfolios $h$ priced one. Then we look for $h=\left(h_{1}, \ldots h_{n}\right) \in\left(L_{+}^{2}\left(\Sigma_{t}\right)\right)^{n}$ so as to solve

$$
\begin{aligned}
\max & \varphi(h) \\
\text { s.t. } & \sum_{i=1}^{n} h_{i} p_{i}^{t}=1 \\
& h_{i} \geq 0 \quad i=1, \ldots, n
\end{aligned}
$$

Definition 2.4. We define the second measure $\mathfrak{M}^{t}$ of the level of arbitrage opportunities as the optimum value achieved in problem $(I V)$.

Note first that $\mathfrak{M}^{t}$ is scalar for every $t$. The consistency of this definition relies on the solvability of Problem $(I V)$. We prove it in Section 3.

\section{Measuring with short sale restrictions}

In this section we prove the solvability of problem $(I V)$, and hence the consistency of $\mathfrak{M}^{t}$. Furthermore, if $h^{*}$ is an optimal solution in problem $(I V)$ and $x^{*}$ is a corresponding optimal solution in problem $\left(I I_{h^{*}}\right)$ we prove that the function $-\sum_{i=1}^{n} x_{i}^{*} p_{i}^{t}$ does not depend on the chosen optimal solutions $h^{*}$ and $x^{*}$. Thus, such a function defines a third measure of the arbitrage opportunities.

From now on, we adopt the following notations: $\mathcal{H}^{t}$ denotes the set $\left\{h \in\left(L_{+}^{2}\left(\Sigma_{t}\right)\right)^{n} \mid \sum_{i=1}^{n} h_{i} p_{i}^{t} \in L^{2}\left(\Sigma_{t}\right)\right\}$ and $F_{h}$ the feasible set in $\left(I I_{h}\right)$.

Lemma 3.1. Problem $\left(I I_{h}\right)$ is solvable for every $h \in \mathcal{H}^{t}$. 
Proof. The proof of the lemma relies on the fact that we do not need to bound the whole feasible set, but only a subset of it containing the optimal solution.

Fix $h \in \mathcal{H}^{t}$. First note that the feasible set $F_{h}$ is non void since $x=0 \in$ $F_{h}$. Furthermore, the value of $\left(I I_{h}\right)$ is bounded by $\sum_{i=1}^{n} \int_{\Omega} h_{i} p_{i}^{t} d P$ and hence finite. Moreover, it is nonnegative.

For every $x \in F_{h}$, let $A=\left\{\omega \in \Omega \mid-\sum_{i=1}^{n} x_{i}(\omega) p_{i}^{t}(\omega)>0\right\}$ and $x^{\prime}=x \chi_{A}$, where $\chi_{A}$ denotes the characteristic function of $A$.

Then, $x^{\prime} \in F_{h}$ and $-\sum_{i=1}^{n} \int_{\Omega} x_{i}^{\prime} p_{i}^{t} d P \geq-\sum_{i=1}^{n} \int_{\Omega} x_{i} p_{i}^{t} d P$. Consequently we can take as feasible set for $\left(I I_{h}\right)$ the subset $F_{h}^{\prime}=\{x \in$ $\left.F_{h} \mid \sum_{i=1}^{n} x_{i}(\omega) p_{i}(\omega) \leq 0\right\}$ of $F_{h} . \quad F_{h}^{\prime}$ is an order bounded set since $-h_{i} \leq x_{i} \leq\left(\sum_{j \neq i} h_{j} p_{j}^{t}\right) / p_{i}^{t}$ whenever $x \in F_{h}^{\prime}$. Thus, $F_{h}^{\prime}$ is a weaklycompact set. Then, the weak-continuity of the objective function guarantees the solvability of Problem $\left(I I_{h}\right)$.

The lemma above allows us to properly define $\varphi(h)$ as the optimal value in $\left(I I_{h}\right)$, that is,

$$
\varphi(h)=\max \left\{-\sum_{i=1}^{n} \int_{\Omega} x_{i} p_{i}^{t} d P \mid x \in F_{h}\right\} .
$$

It is easily verified that

$$
\varphi\left(h+h^{\prime}\right) \geq \varphi(h)+\varphi\left(h^{\prime}\right) \quad \text { and } \quad \varphi(\delta h)=\delta \varphi(h)
$$

for every $h, h^{\prime} \in \mathcal{H}^{t}$ and $\delta>0$, so $\varphi$ is a concave function.

Also, $\varphi(h) \geq \varphi\left(h^{\prime}\right)$ if $h \geq h^{\prime}$ in the usual partial order of $L^{2}\left(\Sigma_{t}\right)$.

Finally, $\varphi(h) \leq \sum_{i=1}^{n} \int_{\Omega} h_{i} p_{i}^{t} d P$ since $-\sum_{i=1}^{n} \int_{\Omega} x_{i} p_{i}^{t} d P \leq \sum_{i=1}^{n} \int_{\Omega} h_{i} p_{i}^{t} d P$ for every feasible $x$.

Lemma 3.2. Suppose that $x \in\left(L^{2}\left(\Sigma_{t}\right)\right)^{n}$ is an arbitrage opportunity and let be $A \in \Sigma_{t}$ with $P(A)>0$ and such that $\sum_{i=1}^{n} x_{i} p_{i}^{t+1} \geq 0$ a.e. in $A$ and $\sum_{i=1}^{n} x_{i} p_{i}^{t}<0$ a.e. in $A$. Then for every $\varepsilon>0$ there exists $B \in \Sigma_{t}, B \subseteq A$ with $P(A-B)<\varepsilon$ such that $\hat{x}=x \cdot \chi_{B} \in\left(L^{\infty}\left(\Sigma_{t}\right)\right)^{n}, \sum_{i=1}^{n} \hat{x}_{i} p_{i}^{t+1} \geq 0$ and $\sum_{i=1}^{n} \hat{x}_{i} p_{i}^{t}<0$ a.e. in $B$.

Proof. For every $k \in \mathbb{N}$ take $A_{k}=\left\{\omega \in A|| x_{i}(\omega) \mid \leq k \quad i=1,2 \ldots, n\right\}$. Since $\lim _{k} P\left(A_{k}\right)=P(A)$, then for every $\varepsilon>0$, there exists $k^{\prime} \in \mathbb{N}$ such 
that $P\left(A-A_{k}\right)<\varepsilon$ whenever $k \geq k^{\prime}$. Then $B=A_{k^{\prime}}$ is the required subset.

Proposition 3.1. No arbitrage opportunity exists at date $t$ if and only if $\varphi(h)=0$ for every $h \in \mathcal{H}^{t}$.

Proof. Suppose there exists an arbitrage portfolio $x \in\left(L^{2}\left(\Sigma_{t}\right)\right)^{n}$ and let $A \in \Sigma_{t}$ such that $P(A)>0, \sum_{i=1}^{n} x_{i} p_{i}^{t+1} \geq 0$ a.e. in $A$ and $\sum_{i=1}^{n} x_{i} p_{i}^{t}<0$ a.e. in $A$.

From Lemma 3.2, let be $B$ and

$$
\tilde{x}(\omega)= \begin{cases}x & \text { if } \omega \in B \\ 0 & \text { otherwise }\end{cases}
$$

such that $P(B)>0$ and $\tilde{x} \in\left(L^{\infty}\left(\Sigma_{t}\right)\right)^{n}$. Hence, $\sum_{i=1}^{n}\left|\tilde{x}_{i}\right| p_{i}^{t} \in L^{2}\left(\sum_{t+1}\right)$. Set $h=\left(\left|\tilde{x}_{1}\right|, \ldots,\left|\tilde{x}_{n}\right|\right)$. Then, $\tilde{x} \in F_{h}$ and consequently

$$
\varphi(h) \geq-\sum_{i=1}^{n} \int_{\Omega} \tilde{x}_{i} p_{i}^{t} d P=-\sum_{i=1}^{n} \int_{B} x_{i} p_{i}^{t} d P>0
$$

Assume now that no arbitrage opportunity exists. Then for every $h \in$ $\left(L_{+}^{2}\left(\Sigma_{t}\right)\right)^{n}$ and $x \in F_{h}$ one has that,

$$
P\left\{\omega \mid \sum_{1=1}^{n} x_{i} p_{i}^{t}<0\right\}=0 .
$$

Consequently $\varphi(h)=0$ for every $h \in \mathcal{H}^{t}$.

In order to prove that Problem $(I V)$ is solvable, we introduce the following optimization program,

$$
\begin{aligned}
\max - & \sum_{i=1}^{n} \int_{\Omega} x_{i} p_{i}^{t} d P \\
\text { s.t. } & \sum_{i=1}^{n} x_{i} p_{i}^{t+1} \geq 0 \\
& \sum_{i=1}^{n} h_{i} p_{i}^{t}=1 \\
& x_{i} \geq-h_{i} \quad i=1, \ldots, n \\
& x_{i} \in L^{2}\left(\Sigma^{t}\right), \quad h_{i} \in L_{+}^{2}\left(\Sigma^{t}\right) \quad i=1, \ldots, n
\end{aligned}
$$


Note that Problem $(V)$ is equivalent to the combined problem from $\left(I I_{h}\right)$ and $(I V)$. Furthermore, Problem $(V)$ is just a linear program where the decision variable $(x, h)$ takes values in $\left(L^{2}\left(\Sigma_{t}\right)\right)^{n} \times\left(L_{+}^{2}\left(\Sigma_{t}\right)\right)^{n}$.

Theorem 3.1. Problem $(V)$ is solvable. Consequently, Problem $(I V)$ is also solvable.

Proof. Just proceed as in the proof of Lemma 3.1 to choose a subset $F$ of the feasible set of $V$, such that $(-1) / p_{i} \leq-h_{i} \leq x_{i} \leq 1 / p_{i}$ whenever $(x, h) \in F$. Then Problem $(V)$ is solvable in a such weakly compact feasible region. However, the choice of $F$ yields an optimal solution for Problem $(V)$ in the whole feasible set.

Finally, note that an optimal solution $\left(x^{*}, h^{*}\right)$ of Problem $(V)$ yields an optimal solution $h^{*}$ of Problem $(I V)$ and an optimal solution $x^{*}$ of Problem $\left(I I_{h^{*}}\right)$.

Remark 3.1. In empirical applications an optimal solution of $(I V)$ may be obtained by solving $(V)$ (see Balbás et al. (1999), Balbás et al. (2000), or Pardo et al. (2002), where it is illustrated how the Balbás and Muñoz-Bouzo (1998) static measure may be computed in empirical analysis). Problem $(V)$ is just a linear program which can be solved by the classical optimization techniques (see Anderson and Nash (1987)). Furthermore, in some specially interesting situations, the $\sigma$-algebras $\Sigma_{t}$ and $\Sigma_{t+1}$ will be finite and then $(V)$ can be solved by the simplex method.

Remark 3.2. It can be deduced from the proof of Theorem 3.1 that an optimal solution $\left(x^{*}, h^{*}\right)$ of Problem $(V)$ verifies

$$
(-1) / p_{i} \leq-h_{i}^{*} \leq x_{i}^{*} \leq 1 / p_{i} .
$$

Thus, assuming $A_{1}$, one gets that $\left(x^{*}, h^{*}\right) \in\left(L^{\infty}\left(\Sigma_{t}\right)\right)^{2 n}$. So, programs $\left(I I_{h}\right),(I V)$ and $(V)$ can be reformulated in such a way that all the spaces involved are $L^{\infty}$-spaces.

Remark 3.3. Finally, it should be noted that assumption $A_{2}$ has not been needed for the proofs of Lemma 3.1 and Theorem 3.1. More precisely, the condition $p_{i}^{t+1} \in L_{+}^{\infty}\left(\Sigma_{t+1}\right)$ can be relaxed to $p_{i}^{t+1} \in L^{2}\left(\Sigma_{t+1}\right)$ without modifying the results about the solvability of programs $\left(I I_{h}\right),(I V)$ and $(V)$. The condition $p_{i}^{t+1} \in L_{+}^{\infty}\left(\Sigma_{t+1}\right)$ is only needed for the results obtained in Section 4. 
Recall that we denote by $\mathfrak{M}^{t}$ the optimum value achieved in $(I V)$ (see Definition 2.4 or $(V)$, and therefore, it follows from Proposition 3.1 that $\mathfrak{M}^{t}=0$ if and only if there are no arbitrage portfolios at date $t$.

Our next purpose is to prove that an optimal solution $h^{*}$ which maximizes an attainable expected payment, also maximizes an attainable profit in almost every state of nature.

Let $x^{h} \in F_{h}$ be a solution where the optimum value $\varphi(h)$ is achieved. We first prove that $x^{h}$ solves a multiobjective optimization problem. For every $A \in \Sigma_{t}$ consider the restricted optimization problems:

$$
\begin{aligned}
\max & -\sum_{i=1}^{n} \int_{A} x_{i} p_{i}^{t} d P \\
\text { s.t. } & \sum_{i=1}^{n} x_{i} p_{i}^{t+1} \geq 0 \quad \text { a.e. in } \mathrm{A} \\
& x_{i} \geq-h_{i} \quad \text { a.e. in } \mathrm{A}, i=1, \ldots, n
\end{aligned}
$$

Lemma 3.3. Suppose that $x^{h}$ solves $\left(I I_{h}\right)$ for $h \in \mathcal{H}^{t}$. Then, $x^{h}$ solves $\left(I I_{h}^{A}\right)$ for every $A \in \Sigma_{t}$.

Proof. Proceeding by contradiction suppose there exists $x$ feasible in $\left(I I_{h}^{A}\right)$ such that

$$
-\sum_{i=1}^{n} \int_{A} x_{i} p_{i}^{t} d P>-\sum_{i=1}^{n} \int_{A} x_{i}^{h} p_{i}^{t} d P
$$

Define

$$
x^{\prime}= \begin{cases}x^{h} & \text { if } \omega \notin A \\ x & \text { if } \omega \in A .\end{cases}
$$

Obviously $x^{\prime}$ is feasible in $\left(I I_{h}\right)$ and $-\sum_{i=1}^{n} \int_{\Omega} x_{i} p_{i}^{t} d P>\varphi(h)$ and the proof is concluded.

Theorem 3.2. Suppose that $x^{h}$ solves $\left(I I_{h}\right)$ for $h \in \mathcal{H}^{t}$. 
Then $x^{h}$ solves the following vector optimization program:

$$
\begin{aligned}
& \max -\sum_{i=1}^{n} x_{i} p_{i}^{t} \\
& \text { s.t. } \sum_{i=1}^{n} x_{i} p_{i}^{t+1} \geq 0 \\
& \quad x_{i} \geq-h_{i} \quad i=1, \ldots, n
\end{aligned}
$$

Furthermore, $-\sum_{i=1}^{n} x_{i}^{h} p_{i}^{t}$ is a strong maximum in $\left(V I_{h}\right)$, i.e., an upper bound for $\left(V I_{h}\right)$, and not only a maximal value.

Proof. It follows immediately from Lemma 3.3.

Theorem above allows us to define for every $h \in \mathcal{H}^{t}$ a function $\Upsilon$ of the maximum arbitrage profit obtained by an agent holding a portfolio $h$. Concretely $\Upsilon(h, \quad)$ is the strong maximum in $\left(V I_{h}\right)$ and

$$
\Upsilon(h, \omega)=-\sum_{i=1}^{n} x_{i}^{h}(\omega) p_{i}^{t}(\omega)
$$

From $x_{i}^{h} \geq-h_{i}$ it follows that $\Upsilon(h, \quad)$ takes values in $L^{2}\left(\Sigma_{t}\right)$ whenever $h \in \mathcal{H}^{t}$.

Hereafter, we denote by $H^{t}$ the feasible set in $(I V)$, i.e.,

$$
H^{t}=\left\{h \in \mathcal{H}^{t} \mid \sum_{i=1}^{n} h_{i} p_{i}^{t}=1\right\} .
$$

It follows from $A_{1}$ that $H^{t}$ is a subset of $\left(L^{\infty}\left(\Sigma_{t}\right)\right)^{n}$.

Theorem 3.3. Let $h^{*} \in H^{t}$ such that $\mathfrak{M}^{t}=\varphi\left(h^{*}\right)$. Then, $h^{*}$ solves the following vector optimization problem

$$
\max \Upsilon(h, \quad) \quad \text { s.t. } h \in H^{t}
$$

Furthermore, $\Upsilon\left(h^{*}, \quad\right)$ is a strong maximum in (VII). 
Proof. Let $x^{*} \in F_{h^{*}}$ such that $\varphi\left(h^{*}\right)=-\sum_{i=1}^{n} \int_{\Omega} p_{i}^{t} x_{i}^{*} d P$. From Theorem 3.2 we get that

$$
\Upsilon\left(h^{*}, \omega\right)=-\sum_{i=1}^{n} x_{i}^{*}(\omega) p_{i}^{t}(\omega)
$$

Proceeding by contradiction suppose that $\Upsilon\left(h^{*}, \quad\right)$ is not a strong maximum in $(V I I)$. Then, there exist $\hat{h} \in H^{t}, \hat{x} \in F_{\hat{h}}$ and $A \in \Sigma_{t}$ with $P(A)>0$ such that

$$
-\sum_{i=1}^{n} \hat{x}_{i}(\omega) p_{i}^{t}(\omega)=\Upsilon(\hat{h}, \omega)>\Upsilon\left(h^{*}, \omega\right)
$$

for every $\omega \in A$. Setting,

$$
x_{i}(\omega)=\left\{\begin{array}{ll}
\hat{x}_{i}(\omega) & \text { if } \omega \in A \\
x_{i}^{*}(\omega) & \text { otherwise }
\end{array} \text { and } \quad h_{i}(\omega)= \begin{cases}\hat{h}_{i}(\omega) & \text { if } \omega \in A \\
h_{i}^{*}(\omega) & \text { otherwise }\end{cases}\right.
$$

then, $h$ is feasible in $(I V)$ and $x \in F_{h}$ and consequently,

$$
\begin{aligned}
\varphi(h) \geq-\sum_{i=1}^{n} \int_{\Omega} x_{i} p_{i}^{t} d P=-\sum_{i=1}^{n} \int_{A} x_{i} p_{i}^{t} d P & -\sum_{i=1}^{n} \int_{\Omega-A} x_{i} p_{i}^{t} d P> \\
& >-\sum_{i=1}^{n} \int_{\Omega} x_{i}^{*} p_{i}^{t} d P=\varphi\left(h^{*}\right)
\end{aligned}
$$

This strict inequality is in contradiction with the choice of $h^{*}$ as a solution in problem $(I V)$.

Theorem 3.3 shows that $-\sum_{i=1}^{n} x_{i}^{*} p_{i}^{t+1}$ does not depend on the optimal solution $\left(x^{*}, h^{*}\right)$ of $(V)$ and consequently, leads to the following definition.

Definition 3.1. The measure $m^{t}$ of the level of arbitrage opportunities is defined by

$$
m^{t}(\omega)=-\sum_{i=1}^{n} x_{i}^{*}(\omega) p_{i}^{t}(\omega)=\Upsilon\left(h^{*}, \omega\right)
$$

where $h^{*} \in H^{t}$, and $x^{*} \in F_{h^{*}}$ are such that

$$
\mathfrak{M}^{t}=\varphi\left(h^{*}\right)=-\sum_{i=1}^{n} \int_{\Omega} x_{i}^{*} p_{i}^{t} d P .
$$


hence $m^{t} \in L^{2}\left(\Sigma_{t}\right)$.

Observe that $0 \leq m^{t}(\omega)=-\sum_{i=1}^{n} x_{i}^{*}(\omega) p_{i}^{t}(\omega) \leq \sum_{i=1}^{n} h_{i}^{*}(\omega) p_{i}^{t}(\omega)=1$ and

The measure $m^{t}$, therefore, reflects in almost every state of nature the maximum attainable profit from an arbitrage opportunity, obtained among all the investors holding a priced one portfolio. Theorem 3.3 shows that $m^{t}$ is obtained as a strong optimum in a multiobjective optimization program (Problem $(V I I))$.

One can check that, with this definition, $m^{t}$ verifies the first requirement to be a measure of the level of arbitrage opportunities, that is,

Theorem 3.4. The following conditions are equivalent.

i) No arbitrage opportunity exists on the market.

ii) $\mathfrak{M}^{t}=0$.

iii) $m^{t}=0$.

The nonexistence of arbitrage opportunities is thus made testable by estimating $m^{t}$ directly. The closer the value of $\mathfrak{M}^{t}=\int_{\Omega} m^{t} d P$ to zero, the lower the maximum expected quotient between the profit and the total price of short-selling restrictions.

Such a test is also valid for a measurement of market integration: for two or more not integrated markets, treated as parts of one combined market, $\mathfrak{M}^{t}$ also indicates the level of arbitrage opportunities across the markets. More concretely, take as given two markets $A$ and $B$. Then, we treat them as parts of one combined market $C$. We compute $m^{t}$ and $\mathfrak{M}^{t}$ on the global market $C$ to size the level of integration of both markets. However, the maximizing profits given by $m^{t}$ and $\mathfrak{M}^{t}$ englobe the profits derived from the cross-market arbitrage and also the profits derived from the arbitrage opportunities in each market separately. Hence, in order to capture which part of these profits comes from the cross-market arbitrage opportunities , we can consider the measures $m^{t}-\max \left(m_{A}^{t}, m_{B}^{t}\right)$ and $\mathfrak{M}^{t}-\max \left(\mathfrak{M}_{A}^{t}, \mathfrak{M}_{B}^{t}\right)$ whenever there are arbitrage opportunities on $A$ or $B$ (or both). Balbás et al. (2000) and Pardo el al. (2002) illustrate how to implement these techniques when dealing with static models and the measure of Balbás and Muñoz-Bouzo (1998). 


\section{The state prices and the dual approach}

In this section we turn our attention to the dual problem $\left(I I I_{h}\right)$. Before starting our discussion of duality theory we shall study some properties of the dual problem $\left(I I I_{h}\right)$.

Problem $\left(I I I_{h}\right)$ is consistent since $(f, l)=(0, p)$ is feasible. Furthermore, problem $\left(I I I_{h}\right)$ has a finite value since problem $\left(I I_{h}\right)$ is consistent. Nevertheless, solvability of $\left(I I I_{h}\right)$ presents a problem: its feasible set is not bounded and solvability cannot be settled by using Alaoglu's theorem. This is easily overcome by re-posing the problem as

$$
\min \sum_{i=1}^{n} \int_{\Omega} h_{i} l_{i} d P \quad \text { s.t. } \quad l \in \bar{\Lambda}
$$

where

$$
\Lambda=\left\{l \in\left(L_{+}^{2}\left(\Sigma_{t}\right)\right)^{n} \mid E\left(f p_{i}^{t+1} \mid \Sigma_{t}\right)+l_{i}=p_{i}^{t}, i=1, \ldots, n, f \in L_{+}^{2}\left(\Sigma_{t+1}\right)\right\}
$$

and $\bar{\Lambda}$ denotes its closure (in any topology consistent with the duality since $\Lambda$ is a convex set). It is easy to prove that $\left(I I I_{h}\right)$ and $\left(V I I I_{h}\right)$ have the same value and Problem $\left(V I I I_{h}\right)$ is solvable since $\bar{\Lambda}$ is a weakly-compact set.

The dual problem also presents an associated vector optimization problem with strong solutions.

Proposition 4.1. Suppose that $l \in \bar{\Lambda}$ solves $\left(V I I I_{h}\right)$. Then, $l$ solves the following optimization problem

$$
\min \sum_{i=1}^{n} h_{i} l_{i} \quad \text { s.t } \quad l \in \bar{\Lambda}
$$

Proof. Proceeding by contradiction suppose there exists $\lambda^{\prime} \in \bar{\Lambda}$ such that $P(A)>0$ where $A=\left\{\omega \in \Omega \mid \sum_{i=1}^{n} h_{i}(\omega) \lambda_{i}^{\prime}(\omega)<\sum_{i=1}^{n} h_{i}(\omega) \lambda_{i}(\omega)\right\}$. Take $\bar{\lambda}=\lambda^{\prime} \cdot \chi_{A}+\lambda \cdot \chi_{\Omega-A}$. It is easily proved that $\bar{\lambda} \in \bar{\Lambda}$. Besides, the inequality

$$
\sum_{i=1}^{n} \int_{\Omega} h_{i} \bar{\lambda}_{i} d P=\sum_{i=1}^{n} \int_{A} h_{i} \lambda_{i}^{\prime} d P+\sum_{i=1}^{n} \int_{\Omega-A} h_{i} \lambda_{i} d P<\sum_{i=1}^{n} \int_{\Omega} h_{i} \lambda_{i} d P
$$

holds and yields a contradiction. 
In particular, Proposition 4.1 states that an optimal solution of $\left(V I I I_{h}\right)$ also solves the restricted program to every $A \in \Sigma_{t}$, that is,

$$
\min \sum_{i=1}^{n} \int_{A} h_{i} l_{i} d P \quad \text { s.t. } \quad l \in \overline{\Lambda_{A}}
$$

where

$$
\begin{aligned}
\Lambda_{A}=\left\{l \in\left(L_{+}^{2}\left(\Sigma_{t} \cap A\right)\right)^{n} \mid E\left(f p_{i}^{t+1} \mid \Sigma_{t} \cap A\right)+l_{i}=p_{i}^{t}\right. \\
\\
\left.\operatorname{in} A, i=1, \ldots, n, f \in L_{+}^{2}\left(\Sigma_{t+1}\right)\right\} .
\end{aligned}
$$

From the weak-duality relation between $\left(I I_{h}^{A}\right)$ and $\left(I I I_{h}^{A}\right)$ one gets that

$$
-\sum_{i=1}^{n} \int_{A} x_{i} p_{i}^{t} d P \leq \sum_{i=1}^{n} \int_{A} l_{i} h_{i} d P
$$

for every $A \in \Sigma_{t}$ and for every $l \in \Lambda$. Then, this inequality remains true for every $l \in \bar{\Lambda}$. Consequently a weak-duality relation can be stated for the associated vector optimization problems. More precisely, one has that

$$
-\sum_{i=1}^{n} x_{i} p_{i}^{t} \leq \sum_{i=1}^{n} l_{i} h_{i}
$$

for every $x$ feasible in $\left(I I_{h}\right)$ and for every $l \in \bar{\Lambda}$.

For a finite linear program the values of the primal and dual programs are always equal. This is not the case for infinite-dimensional linear programs. The usual conditions under which this property holds do not apply here. This leads us to assume in the remainder of this section the nonexistence of duality gap for $\left(I I_{h}\right)$ and its dual $\left(I I I_{h}\right)$. To motivate this assumption, we begin by proving that there is no duality gap when the $\sigma$-algebra $\Sigma_{t}$ is generated by a finite or countable partition of $\Omega$. This is an important case since in particular it contains most of the practical situations where $\Sigma_{t}$ can be thought of as a finite $\sigma$-algebra.

Theorem 4.1. Suppose that $A_{0}, A_{1}, \ldots$ is a finite or countable partition of $\Omega$ generating the $\sigma$-algebra $\Sigma_{t}$. Then, there is no duality gap for $\left(I I_{h}\right)$ and $\left(I I I_{h}\right)$. 
Proof. Let us prove that there is no duality gap for $\left(I I_{h}\right)$ and $\left(V I I I_{h}\right)$. Consider the restricted optimization programs to each $A_{j}$. Note that every $\Sigma_{t}$-measurable function must have some constant value over $A_{j}$. In particular, $p_{i}^{t}, h_{i}$ have a constant value over $A_{j}$, say $p_{i}^{j}$ and $h_{i}^{j}$. The restricted problems to each $A_{j}$ can be posed as

$$
\begin{aligned}
& \max -\sum_{i=1}^{n} x_{i} p_{i}^{j} \\
& \text { s.t. } \sum_{i=1}^{n} x_{i} p_{i}^{t+1} \geq 0 \quad \text { a.e in } A_{j} \\
& x_{i} \geq-h_{i}^{j} \quad i=1, \ldots, n \\
& x_{i} \in \mathbb{R} \quad i=1, \ldots, n
\end{aligned}
$$

and

$$
\min \sum_{i=1}^{n} h_{i}^{j} l_{i} \quad \text { s.t. } \quad l \in \overline{\Lambda_{j}}
$$

where

$$
\Lambda_{j}=\left\{l \in \mathbb{R}_{+}^{n} \mid \int_{A_{j}} f p_{i}^{t+1} d P+l_{i}=p_{i}^{j}, i=1, \ldots, n, f \in L_{+}^{2}\left(\Sigma_{t+1}\right)\right\} .
$$

In the inequality-constrained program $\left(I I_{h}^{j}\right)$ the associated positive cone $P$ is $\mathbb{R}^{n}$ while the inequality constraints take values in $L^{2}\left(\Sigma_{t+1}\right) \times \mathbb{R}^{n}$, where the associated positive cone $Q$ is $L_{+}^{2}\left(\Sigma_{t+1}\right) \times \mathbb{R}_{+}^{n}$.

$P$ is a cone with compact sole (since $B=\left\{x \in \mathbb{R}^{n} \mid\|x\|=1\right\}$ is a compact set in $P$ such that 0 is not in $B$ and $B$ spans $P$ ). Besides, if $x \in \mathbb{R}$ is such that $\left(\sum_{i=1}^{n} x_{i} p_{i}^{t+1}, x\right) \in Q$ and $-\sum_{i=1}^{n} x_{i} p_{i}^{j}=0$, then $x=0$.

These two facts allow us to ensure that

$$
D^{\prime}=\left\{\left(\sum_{i=1}^{n} x_{i} p_{i}^{t+1}, x-y, \sum_{i=1}^{n} x_{i} p_{i}^{j}\right) \mid x \in \mathbb{R}^{n}, y \in \mathbb{R}_{+}^{n}\right\}
$$

is a closed set (see Theorem 3.19 of Anderson and Nash (1987)). Now, from Theorem 3.10 of Anderson and Nash (1987) it can be deduced that for every $j \in \mathbb{N}$ there exists $l^{j} \in \overline{\Lambda_{j}}$ and $x^{j}$ feasible in $\left(I I_{h}^{j}\right)$ such that

$$
-\sum_{i=1}^{n} p_{i}^{j} x_{i}^{j}=\sum_{i=1}^{n} h_{i}^{j} l_{i}^{j}
$$


Define now for every $q \in \mathbb{N}$ and every $i=1, \ldots, n$ the $\Sigma_{t}$-measurable functions,

$$
x_{i}^{(q)}(\omega)= \begin{cases}x_{i}^{j} & \text { if } \omega \in A_{j} \text { and } j \leq q \\ 0 & \text { otherwise }\end{cases}
$$

and

$$
l_{i}^{(q)}(\omega)= \begin{cases}l_{i}^{j} & \text { if } \omega \in A_{j} \text { and } j \leq q \\ p_{i}^{t}(\omega) & \text { otherwise }\end{cases}
$$

It is easily checked that $x^{(q)}$ is feasible in $\left(I I_{h}\right)$ and $l^{(q)} \in \bar{\Lambda}$. Besides, denoting by $A^{q}=\bigcup_{j=1}^{q} A_{j}$, one has that the relation

$$
\begin{aligned}
\sum_{i=1}^{n} \int_{\Omega} h_{i} l_{i}^{(q)} d P & =\sum_{i=1}^{n} \int_{A^{q}} h_{i} l_{i}^{(q)} d P+\sum_{i=1}^{n} \int_{\Omega-A^{q}} h_{i} l_{i}^{(q)} d P \\
& =-\sum_{i=1}^{n} \int_{A^{q}} p_{i}^{t} x_{i}^{(q)} d P+\sum_{i=1}^{n} \int_{\Omega-A^{q}} h_{i} p_{i}^{t} d P \\
& =-\sum_{i=1}^{n} \int_{\Omega} p_{i}^{t} x_{i}^{(q)} d P+\sum_{i=1}^{n} \int_{\Omega-A^{q}} h_{i} p_{i}^{t} d P
\end{aligned}
$$

holds for every $q \in \mathbb{N}$. Finally, since $\lim _{q \rightarrow \infty} \sum_{i=1}^{n} \int_{\Omega-A^{q}} h_{i} p_{i}^{t} d P=0$ it follows that $\sum_{i=1}^{n} \int_{\Omega} h_{i} l_{i} d P=-\sum_{i=1}^{n} \int_{\Omega} x_{i} p_{i}^{t} d P$, which concludes the proof of the theorem.

Next theorem ensures that in absence of duality gap for $\left(I I_{h}\right)$ and $\left(I I I_{h}\right)$, the stochastic measure $m^{t}$ can be formulated in terms of the optimal solution $\lambda^{*} \in \bar{\Lambda}$ of $\left(V I I I_{h}\right)$.

Theorem 4.2. Assume that there is not duality gap between $\left(I I_{h}\right)$ and $\left(I I I_{h}\right)$ for every $h \in H^{t}$. Then, the equality $-\sum_{i=1}^{n} x_{i}^{h} p_{i}^{t}=\sum_{i=1}^{n} l_{i} h_{i}$ holds whenever $x^{h}$ solves $\left(I I_{h}\right)$ and l solves $\left(V I I I_{h}\right)$. In particular, one has that

$$
m^{t}(\omega)=-\sum_{i=1}^{n} x_{i}^{*}(\omega) p_{i}^{t}(\omega)=\sum_{i=1}^{n} l_{i}^{*}(\omega) h_{i}^{*}(\omega)
$$

where $h^{*} \in H^{t}, x^{*} \in F_{h^{*}}$ are as in Definition 5 and $l^{*} \in \bar{\Lambda}$ solves Problem $\left(V I I I_{h^{*}}\right)$.

Proof. Just apply Proposition 4.1 and the weak-duality relation stated for the vector optimization problems $\left(V I_{h}\right)$ and $\left(V I I I_{h}\right)$. 
The latter theorem points out that the optimization problems introduced in this paper have very special and, in some sense surprising properties. First, both problems, $\left(V I_{h}\right)$ and $\left(I X_{h}\right)$, have a strong solution. Second, we can conclude that there is no duality gap for $\left(V I_{h}\right)$ and $\left(I X_{h}\right)$ whenever there is no duality gap for the scalar problems $\left(I I_{h}\right)$ and $\left(I I I_{h}\right)$. However, $\left(I X_{h}\right)$ is not the dual problem of $\left(V I_{h}\right)$ since its dual variables must be an element of the space of all bounded linear operators from $L^{2}\left(\Sigma_{t}\right)$ into itself (see for instance Sawaragi et al. (1985), Kuk et al. (1996) or Balbás and Guerra (1996)). Furthermore, the absence of duality gap for scalarized dual vector problems does not guarantee in general the absence of duality gap for the vector problems. This is not our case even considering that in $\left(I X_{h}\right)$ the feasible set is a strong simplification (and then a subset) of the feasible set of the dual of $\left(V I_{h}\right)$. Finally, since Problem $\left(I X_{h}\right)$ also has a strong solution, the primal sensitivity (given by $l$ ) can be easily studied, what would be far more difficult without strong solutions (see the references above).

In absence of duality gap, $\phi(h)$, the optimal value of $\left(I I_{h}\right)$, can be obtained as

$$
\phi(h)=\inf _{\lambda \in \Lambda} \sum_{i=1}^{n} \int_{\Omega} h_{i} \lambda_{i} d P=\min _{\lambda \in \bar{\Lambda}} \sum_{i=1}^{n} \int_{\Omega} h_{i} \lambda_{i} d P
$$

Hence, the problem of finding $\mathfrak{M}^{t}$ can be expressed by a max-min problem

$$
\mathfrak{M}^{t}=\max _{h \in H^{t}} \inf _{\lambda \in \Lambda} U(\lambda, h),
$$

where $U$ is defined by $U(\lambda, h)=\sum_{i=1}^{n} \int_{\Omega} h_{i} \lambda_{i} d P$. A min-max theorem is now established.

Theorem 4.3. The equality $\max _{h \in H^{t}} \inf _{l \in \Lambda} U(l, h)=\inf _{l \in \Lambda} \max _{h \in H} U(l, h)$ holds.

Proof. Note that $H^{t}$ and $\Lambda$ are convex subsets, $H^{t}$ is a weak-compact set, $U(l,$.$) is quasiconcave and weakly upper-semicontinuous for every l \in \Lambda$, and $U(., h)$ is quasiconvex and weakly below-semicontinuous for every $h \in H$. Now, just proceed as in Sion's theorem (Moulin (1979)) to get the result. 
In absence of duality gap for $\left(I I_{h}\right)$ and $\left(I I I_{h}\right)$ for every $h \in H^{t}$, the above theorem states that

$$
\mathfrak{M}^{t}=\max _{h \in H^{t}} \inf _{l \in \Lambda} U(l, h)=\inf _{l \in \Lambda} \max _{h \in H^{t}} U(l, h) .
$$

In game theoretic terminology latter equality expresses a two-person zerosum game of the investor against the "market". Since $l_{i}=p_{i}^{t}-E\left(f p_{i}^{t+1} \mid \Sigma_{t}\right)$ could be interpreted as the error committed by the "market" in the price of each asset for the state prices $f$, the sum $\sum_{i=1}^{n} \int_{\Omega} h_{i} l_{i}$ would be the expected payment from the "market" to the investor due to $h$ and $l$. Thus, the investor chooses a priced one portfolio of short-selling bounds in such a way that it maximizes the minimal expected payment desired by the "market" and solves $\max _{h \in H^{t}} \inf _{l \in \Lambda} U(l, h)$. The problem, $\inf _{l \in \Lambda} \max _{h \in H^{t}} U(l, h)$ describes the process by which the "market" counteracts the goal of the investor by choosing the feasible $l$ which minimizes the maximal expected payment desired by the investor.

Finally note that, under the assumptions of Theorem 4.2, we can also conclude that there is no duality gap for the scalar problem $(V)$ and its dual,

$$
\begin{array}{ll}
\min & \int_{\Omega} \alpha(\omega) d P \\
\text { s.t. } & E\left(f p_{i}^{t+1} \mid \Sigma^{t}\right)+l_{i}=p_{i}^{t} \quad i=1, \ldots, n \\
& \alpha p_{i}^{t} \geq l_{i} \quad i=1, \ldots, n \\
& \alpha \in L_{+}^{2}\left(\Sigma^{t}\right), \\
& l_{i} \in L_{+}^{2}\left(\Sigma^{t}\right) \quad f \in \\
& L_{+}^{2}\left(\Sigma^{t+1}\right) \quad i=1, \ldots, n
\end{array}
$$

as well as there is no duality gap for the corresponding vector problems. Thus, for a feasible $f$, consider it as a possible proxy for discount factors, $E\left(f p_{i}^{t+1} \mid \Sigma^{t}\right)$ becomes the "right price" of the $i^{t h}$-security, $\frac{p_{i}^{t}-E\left(f p_{i}^{t+1} \mid \Sigma^{t}\right)}{p_{i}^{t}}$ the relative (per dollar) "committed error" and $\alpha$ is their maximum. So, latter problem and its corresponding vector problem try to find discount factors in order to minimize the expected, respectively random variable, "maximum relative committed error". 


\section{$5 \quad$ Measuring without short sale restrictions}

The purpose of this section is to prove that the measure $m^{t}$ tests different relative arbitrage profits in almost every state of nature without short selling restrictions. Of special interest is the equality between the maximum expected profit with short-selling restrictions with total price one at the first date of the period and the maximum arbitrage profit obtained relative to the price of the sold assets.

From now on, denote by $\Omega_{1}=\left\{\omega \in \Omega \mid m^{t}(\omega) \neq 0\right\}$ and by $\varphi_{A}(h)$ the optimal value attained in $\left(I I_{h}^{A}\right)$ for every $A \in \Sigma_{t}$. Note that from Theorem 3.3 one can deduce that an optimal solution $h^{*}$ also maximizes $\varphi_{A}$ in $H_{A}^{t}=\left\{h \in\left(L_{+}^{2}\left(\Sigma_{t}\right)\right)^{n} \mid \sum_{i=1}^{n} h_{i} p_{i}^{t}=1\right.$ a.e. in $\left.A\right\}$.

Lemma 5.1. Let $h^{*} \in H^{t}$ and $x^{*} \in F_{h^{*}}$ such that

$$
\mathfrak{M}^{t}=\varphi\left(h^{*}\right)=-\sum_{i=1}^{n} \int_{\Omega} x_{i}^{*} p_{i}^{t} d P
$$

Then,

$$
P\left(\left\{\omega \in \Omega_{1} \mid x_{i}^{*}(\omega)>-h_{i}^{*}(\omega) \neq 0\right\}\right)=0
$$

for every $i=1, \ldots, n$

Proof. In order to simplify the notation and without loss of generality, we will prove for $i=n$.

Proceeding by contradiction, suppose that $P\left(\left\{\omega \in \Omega_{1} \mid x_{n}^{*}(\omega)>-h_{n}^{*}(\omega) \neq\right.\right.$ $0\})>0$. Then, setting $A=\left\{\omega \in \Omega_{1} \mid h_{n}^{*}(\omega) \neq 0\right\}, B=\left\{\omega \in A \mid x_{n}^{*}(\omega) \geq 0\right\}$ and $C=\left\{\omega \in A \mid 0>x_{n}^{*}(\omega)>-h_{n}^{*}(\omega)\right\}$, one has $P(B)>0$ or $P(C)>0$ and $B, C \subseteq \Omega_{1}$.

First assume that $P(B)>0$, and let $h_{0}=\left(h_{1}^{*}, \ldots, h_{n-1}^{*}, 0\right)$ and $\gamma=$ $\sum_{i=1}^{n-1} p_{i}^{t} h_{i}^{*}$

The inequality $\varphi_{B}\left(h_{0}\right) \leq \varphi_{B}\left(h^{*}\right)$ holds, since the feasible set of $\left(I I_{h_{0}}^{B}\right)$ is a subset of the feasible set of $\left(I I_{h^{*}}^{B}\right)$.

Since $x^{*}$ is feasible in $\left(I I_{h_{0}}^{B}\right)$ we get that $\varphi_{B}\left(h^{*}\right)=\varphi_{B}\left(h_{0}\right)$.

Besides, $P(E)=0$ holds, where $E=\left\{\omega \in B \mid p_{n}^{t}(\omega) h_{n}^{*}(\omega)=1\right\}$, since $\varphi_{E}\left(h_{0}\right)=\varphi_{E}\left(h^{*}\right)=\int_{\Omega} m^{t} d P=0$ and $E \subseteq \Omega_{1}$. Then, $\gamma>0$ in $B$. Taking 
into account that $h_{n}^{*}>0$ and $p_{n}^{t}>0$ in $B$, we get $\gamma<1$ in $B$. Then, there exists $D \subseteq B, D \in \Sigma_{t}$ such that $P(D)>0$ and $0<b<\gamma(\omega)<a<1$ in $D$ and consequently $\frac{1}{\gamma} \in L^{\infty}\left(\Sigma_{t}\right)$ and hence $\frac{1}{\gamma} h_{0} \in\left(L_{+}^{2}\left(\Sigma_{t}\right)\right)^{n}$. Finally, we have

$$
\varphi_{D}\left(h^{*}\right)=\varphi_{D}\left(h_{0}\right)=a \varphi_{D}\left(\frac{1}{a} h_{0}\right)<\varphi_{D}\left(\frac{1}{a} h_{0}\right) \leq \varphi_{D}\left(\frac{1}{\gamma} h_{0}\right) \leq \varphi_{D}\left(h^{*}\right)
$$

where the last inequality follows from the fact that $\frac{1}{\gamma} h_{0} \in H_{D}^{t}$. Since (5.1) leads to a contradiction, we get $P(B)=0$.

Assume now that $P(C)>0$ and let $h^{0}=\left(h_{1}^{*}, \ldots, h_{n-1}^{*},\left|x_{n}^{*}\right|\right)$ and $\delta=$ $\sum_{i=1}^{n-1} p_{i}^{t} h_{i}^{*}+p_{n}^{t}\left|x_{n}^{*}\right|$. As above, we get $0<\delta<1$ and $G \subseteq C, G \in \Sigma_{t}$ such that $P(G)>0$ and $0<b<\delta(\omega)<a<1$ in $G$ and consequently $\frac{1}{\delta} \in L^{\infty}\left(\Sigma_{t}\right)$ and hence $\frac{1}{\delta} h_{0} \in\left(L_{+}^{2}\left(\Sigma_{t}\right)\right)^{n}$ : Thus, we derive that

$$
\varphi_{G}\left(h^{*}\right)=\varphi_{G}\left(h^{0}\right)=a \varphi_{G}\left(\frac{1}{a} h^{0}\right)<\varphi_{G}\left(\frac{1}{a} h^{0}\right) \leq \varphi_{G}\left(\frac{1}{\delta} h^{0}\right) \leq \varphi_{G}\left(h^{*}\right)
$$

and we get again a contradiction.

Lemma 5.1 says basically that the portfolio where the maximum expected profit is achieved either sells all the stock or purchases in each asset.

Given a portfolio $x \in\left(L^{2}\left(\Sigma_{t}\right)\right)^{n}$, we denote by $x_{i}^{-}(\omega)=\max \left(-x_{i}(\omega), 0\right)$ and we define the function

$$
f(x, \omega)=\left\{\begin{array}{lll}
\frac{-\sum_{i=1}^{n} x_{i}(\omega) p_{i}^{t}(\omega)}{\sum_{i=1}^{n} x_{i}^{-}(\omega) p_{i}^{t}(\omega)} & \text { if } \sum_{i=1}^{n} x_{i}^{-}(\omega) p_{i}^{t}(\omega) \neq 0 \\
0 & \text { if } \sum_{i=1}^{n} x_{i}^{-}(\omega) p_{i}^{t}(\omega)=0
\end{array}\right.
$$

If $x$ is an arbitrage opportunity at date $t$ and $A \in \Sigma_{t}$ is as in Definition 2.1 , the function $f(x, \quad)$ is the quotient in $A$ between the profit generated by $x$ and the price of all the sold assets, both computed at date $t$. We now consider the following non differentiable optimization problem

$$
\max f\left(x, \quad \text { s.t } \quad \sum_{i=1}^{n} x_{i}(\omega) p_{i}^{t+1}(\omega) \geq 0\right.
$$


Theorem 5.1. Assume that the market satisfies $A_{1}$ and $A_{2}$ and let $x^{*} \in$ $\left(L^{2}\left(\Sigma_{t}\right)\right)^{n}$ such that $m^{t}(\omega)=-\sum_{i=1}^{n} x_{i}^{*}(\omega) p_{i}^{t}(\omega)$. Then, $x^{*}$ solves problem $(X)$ and $m^{t}(\omega)=f\left(x^{*}, \omega\right)$ almost everywhere.

Proof. We first prove that for every $x$ feasible in $(X)$ one has that $f(x, \omega) \leq$ $m^{t}(\omega)$ a.e. in $\Omega$.

Proceeding by contradiction suppose that $P(D)>0$, where $D=\{\omega \in$ $\left.\Omega \mid f(x, \omega)>m^{t}(\omega)\right\}$. Note that $f(x, \omega) \leq 1$ whenever $\omega \in D$. Set $\varepsilon(\omega)=\sum_{i=1}^{n} x_{i}^{-}(\omega) p_{i}^{t}(\omega)>0$. Then, $\varepsilon>0$ in $D$ since $f(x, \omega) \geq 0$ in $D$ and $\varepsilon \in L^{1}\left(\Sigma_{t}\right)$, from where it is deduced the existence of $C \subseteq D, C \in \Sigma_{t}$ such that $P(C)>0$ and $0<a<\varepsilon(\omega)<b$ in $C$. Consequently, the functions $y_{i}=\frac{1}{\varepsilon} x_{i} \in L^{2}\left(\Sigma_{t}\right)$. Define $h^{\prime}=\left(h_{1}^{\prime}, h_{2}^{\prime} \ldots h_{n}^{\prime}\right)$ by $h_{i}^{\prime}=\sup \left(-y_{i}, 0\right)=y_{i}^{-}$for every $i=1, \ldots, n$.

It is easily verified that $\varphi_{C}\left(h^{\prime}\right) \leq \int_{C} m d P$.

Besides, from $y$ feasible in $\left(I I_{h^{\prime}}^{C}\right)$ we get

$$
\int_{C} f(x, \omega) d P(\omega)=-\int_{C} \sum_{i=1}^{n} p_{i}^{t} y_{i} d P \leq \varphi_{C}\left(h^{\prime}\right) .
$$

Thus, combining both inequalities, we obtain a contradiction. Then, $f(x, \omega) \leq m^{t}(\omega)$ a.e. in $\mathrm{A}$.

Assume now that $x=x^{*}$. Since $m^{t}(\omega)=-\sum_{i=1}^{n} x_{i}^{*}(\omega) p_{i}^{t}(\omega)$, and the fact that $x_{i}^{*}(\omega)=-h_{i}(\omega)$ whenever $i \in S_{x^{*}, \omega}$ and $h_{i}^{*}(\omega)=0$ otherwise $B$ a.e., we get

$$
\sum_{i=1}^{n}\left(x_{i}^{*}\right)^{-}(\omega) p_{i}^{t}(\omega)=\sum_{i=1}^{n} h_{i}^{*}(\omega) p_{i}^{t}(\omega)=1
$$

Consequently, $f\left(x^{*}, \omega\right)=m^{t}(\omega)$

Finally, the following theorem states that the same portfolio $x^{*}$ leading to the measures $\mathfrak{M}^{t}$ and $m^{t}$ also leads to the measure $l^{t}$. 
Theorem 5.2. Let $x^{*} \in\left(L^{2}\left(\Sigma_{t}\right)\right)^{n}$ be such that

$$
m^{t}(\omega)=-\sum_{i=1}^{n} x_{i}^{*}(\omega) p_{i}^{t}(\omega)
$$

Then, $x^{*}$ yields a strong maximum in problem $(I)$ and the equality

$$
l^{t}(\omega)=\frac{m^{t}(\omega)}{2-m^{t}(\omega)}
$$

holds.

Proof. We may assume without loss of generality that $x^{*}(\omega)=0$ whenever $\sum_{i=1}^{n}\left(x_{i}^{*}\right)^{-}(\omega) p_{i}^{t}(\omega)=0$. Manipulating $g$ it is easy to prove that

$$
g(x, \omega)=\frac{f(x, \omega)}{2-f(x, \omega)}
$$

for every $x \in\left(L^{2}\left(\Sigma_{t}\right)\right)^{n}$ and whenever $\sum_{i=1}^{n} x_{i}^{-}(\omega) p_{i}^{t}(\omega) \neq 0$. In particular, $g\left(x^{*}, \omega\right)=f\left(x^{*}, \omega\right) /\left(2-f\left(x^{*}, \omega\right)\right)$ holds for almost every $\omega \in \Omega$. Let $x$ be feasible in problem $(I)$. If $\sum_{i=1}^{n} x_{i}^{-}(\omega) p_{i}^{t}(\omega)=0$ and $x(\omega) \neq 0$, then $g(x, \omega)=-1 \leq g\left(x^{*}, \omega\right)$.

If $\sum_{i=1}^{n} x_{i}^{-}(\omega) p_{i}^{t}(\omega) \neq 0$ then, from Theorem 5.1 the inequality $f(x, \omega) \leq$ $f\left(x^{*}, \omega\right)$ holds. Besides, $\frac{t}{2-t}$ is a increasing continuous function in $(-\infty, 1]$ $\left(f(x, \omega), f\left(x^{*}, \omega\right) \leq 1\right)$. Thus, $g(x, \omega) \leq g\left(x^{*}, \omega\right)$ Finally, the equality $l^{t}(\omega)=m^{t}(\omega) /\left(2-m^{t}(\omega)\right)$ comes from the equality $g\left(x^{*}, \omega\right)=f\left(x^{*}, \omega\right) /(2-$ $\left.f\left(x^{*}, \omega\right)\right)$.

\section{Remarks and conclusions}

\section{Relaxing some assumptions}

It is of interest to ask how the results given here must be modified if the model does not verify some of the assumptions.

As we noticed in Section 2, the assumptions imposed to $p_{1}^{t}(t \in \mathbb{N})$ can be replaced by the assumption that one of the securities always has strictly positive price. We can then measure in the security market model with prices so normalized. Passing from the original to the primed model 
involves only a change in units on the prices. Since these changes do not affect Problems $(I)$ and $X$, the same measures are obtained for both models.

We also noticed in Section 3 (see Remark 3.3 after Theorem 3.1) that we can relax the constraints imposed to $p_{i}^{t+1}$. If we only assume that $p_{i}^{t+1} \in$ $L^{2}\left(\Sigma_{t+1}\right)$ for $i=2, \ldots n$, all the results stated here extend to this new setting except for those derived from the established duality (Section 4).

Suppose now that in $A_{1}$ we relax the constraint $p_{i}^{t}(\omega)>k_{i}>0$ assuming only that $p_{i}^{t}(\omega)>0$.

For every $k \in \mathbb{N}$ denote by

$$
A^{k}=\left\{\omega \in \Omega \mid p_{i}^{t}(\omega)>\frac{1}{k}, i=1, \ldots n\right\}
$$

Obviously, $\lim _{k} P\left(A^{k}\right)=1$.

Set $\left(p_{i}^{t}\right)_{k}=p_{i}^{t} \cdot \chi_{A^{k}}$ and consider the programs $\left(I I_{h}^{k}\right),\left(I I I_{h}^{k}\right)$ and $\left(I V^{k}\right)$ where each $p_{i}^{t}$ is replaced by $\left(p_{i}^{t}\right)_{k}$. Choose corresponding zero valued in $\Omega-A^{k}$ functions $h_{i k}^{*}, l_{i k}^{*}, x_{i k}^{*}$ and $m_{k}^{t}$. It follows from Theorem 3.3 that $m_{j}^{t} \cdot \chi_{A^{k}}=m_{k}^{t}$ for every $j \geq k$. Hence $\left(m_{k}^{t}\right)_{k \in \mathbb{N}}$ is an a.e. pointwise convergent sequence such that $0 \leq m_{k}^{t} \leq 1$. Then,

$$
m^{t}=\lim _{k \in \mathbb{N}} m_{k}^{t} \in L^{2}\left(\Sigma_{t}\right)
$$

Note that $\int_{\Omega} m^{t} d P$ is the optimum value in Program $(I V)$, but this optimum is not necessarily attained in $H^{t}$. Nevertheless, for every $\varepsilon>0$ there exist $A \in \Sigma_{t}$ such that $P(A) \geq 1-\varepsilon, h_{\varepsilon} \in H$ such that Program $(I V)$ restricted to $A$ achieves its optimum $\int_{A} m d P$ in $h_{\varepsilon}$ and $x_{\varepsilon} \in F_{h_{\varepsilon}}$ such that Program $\left(I I_{h_{\varepsilon}}^{A}\right)$ achieves its optimum in $x_{\varepsilon}$.

\section{State prices in a $L^{\infty}$-spaces framework}

The dual approach taken in Section 4 is subject to the absence of duality gap for $\left(I I_{h}\right)$ and $\left(I I I_{h}\right)$. Whenever this absence of duality gap cannot be stated, Problem $\left(I I_{h}\right)$ can be reformulated to avoid this problem.

As already said, see Remark 3.2 after Theorem 3.1, an optimal solution $\left(x^{*}, h^{*}\right)$ leading to $\mathfrak{M}^{t}$ verifies that $\left(x^{*}, h^{*}\right) \in\left(L^{\infty}\left(\Sigma_{t}\right)\right)^{2 n}$. Then, program 
$\left(I I_{h}\right)$ can be reformulated as

$$
\begin{aligned}
\max -\sum_{i=1}^{n} & \int_{\Omega} x_{i} p_{i}^{t} d P \\
\text { s.t. } & \sum_{i=1}^{n} x_{i} p_{i}^{t+1} \geq 0 \\
& x_{i} \geq-h_{i} \quad i=1, \ldots, n \\
& x_{i} \in L^{\infty}\left(\Sigma_{t}\right) \quad i=1, \ldots, n
\end{aligned}
$$

for every $h \in\left(L_{+}^{\infty}\left(\Sigma_{t}\right)\right)^{n}$. In this topological framework, we get its dual problem

$$
\begin{aligned}
\min & \sum_{i=1}^{n} \Gamma_{i}\left(h_{i}\right) \\
& \text { s.t. } \Gamma\left(p_{i}^{t+1}\right)+\Gamma_{i}=p_{i}^{t} \\
& \quad \Gamma \in\left(L^{\infty}\left(\Sigma_{t+1}\right)\right)_{+}^{\prime}, \Gamma_{i} \in\left(L^{\infty}\left(\Sigma_{t}\right)\right)_{+}^{\prime} \quad \text { for every } i=1, \ldots, n
\end{aligned}
$$

where $\left(L^{\infty}\left(\Sigma_{t+1}\right)\right)_{+}^{\prime}$ and $\left(L^{\infty}\left(\Sigma_{t}\right)\right)_{+}^{\prime}$ denote the positive cones of the associated dual Banach spaces. The dual constraints must be understood as equalities in $\left(L^{\infty}\left(\Sigma_{t}\right)\right)_{+}^{\prime}$. More precisely, we identify each $p_{i}^{t}$ and each $\Gamma\left(p_{i}^{t+1}\right)$ with the elements of $\left(L^{\infty}\left(\Sigma_{t}\right)\right)_{+}^{\prime}$ such that $p_{i}^{t}(z)=\int_{\Omega} z p_{i}^{t} d P$ and $\Gamma\left(p_{i}^{t+1}\right)(z)=\Gamma\left(z p_{i}^{t+1}\right)$ for every $z \in L^{\infty}\left(\Sigma_{t}\right)$.

The interest of a such topological framework in which to pose our problems is that the conditions of Lagrange duality theorem (see Luenberger (1969)) hold for $\left(I I_{h}^{\infty}\right)$ and $\left(I I I_{h}^{\infty}\right)$. Consequently, there is no duality gap for $\left(I I_{h}^{\infty}\right)$ and $\left(I I I_{h}^{\infty}\right)$. Moreover, $\left(I I I_{h}^{\infty}\right)$ is solvable.

The absence of duality gap allows us to characterize the absence of arbitrage by the existence of state prices that belong to $\left(L^{\infty}\left(\Sigma_{t+1}\right)\right)^{\prime}$.

Theorem 6.1. No arbitrage opportunity exists at date $t$ if and only if there exists $\Gamma \in\left(L^{\infty}\left(\Sigma_{t+1}\right)\right)_{+}^{\prime}$ such that $\Gamma\left(p_{i}^{t+1}\right)=p_{i}^{t}$ for every $i=1, \ldots, n$.

Proof. From Lemma 3.2 and Proposition 3.1 it follows that no arbitrage opportunity exists if and only if $\varphi(h)=0$ for every $h \in\left(L_{+}^{\infty}\left(\Sigma_{t}\right)\right)^{n}$. From the absence of duality gap between $\left(I I_{h}^{\infty}\right)$ and $\left(I I I_{h}^{\infty}\right)$ we conclude that for every $h \in\left(L_{+}^{\infty}\left(\Sigma_{t}\right)\right)^{n}$ there exists $\left(\Gamma_{i}\right)_{i=1}^{n}$ feasible in $\left(I I I_{h}^{\infty}\right)$ such that 
$\sum_{i=1}^{n} \Gamma_{i}\left(h_{i}\right)=0$. Take an arbitrary interior point $h$ of $\left(L_{+}^{\infty}\left(\Sigma_{t}\right)\right)^{n}$. Then, the associated $\left(\Gamma_{i}\right)_{i=1}^{n}$ verifies that $\Gamma_{i}\left(h_{i}\right)=0$ for every $i=1, \ldots, n$. Consequently, $\Gamma_{i}=0$ since $h_{i}$ is an interior point of $L_{+}^{\infty}\left(\Sigma_{t}\right)$ and $\Gamma_{i} \in\left(L^{\infty}\left(\Sigma_{t}\right)\right)_{+}^{\prime}$. Thus, $\Gamma_{i}=0 i=1, \ldots n$ is feasible in $\left(I I I_{h}^{\infty}\right)$ and Theorem 6.1 is proved.

From the absence of duality gap and solvability for both programs when working in $L^{\infty}$-spaces, not only the absence of arbitrage portfolios can be characterized by state prices or dual variables but also the function $\varphi$ and the measure $\mathfrak{M}^{t}$.

\section{Markets with frictions}

There is an important body of literature on asset pricing under different points of view which takes into account transaction costs, among others, Prisman (1986), Davis et al. (1993), Jouini and Kallal (1995), Toft (1996), Chateauneuf et al. (1996), Pham and Touzi (1999) or De Wagenaere and Wakker (2001). Our aim is to incorporate some of their ideas on the transaction costs (bid-ask spread, taxes,...), to derive how do these transaction costs affect to our measures. We will assume three alternative hypotheses on the transaction costs: linear taxes, bid-ask spreads, and a combination of both.

First we assume that we are in presence of linear taxes depending on the total price of all interchanged assets. More precisely, if an investor trades the portfolio $x$ at date $t$, we assume that the transaction costs are given by $C(x, \omega)=C_{0} \sum_{i=1}^{n}\left|x_{i}^{t}(\omega)\right| p_{i}^{t}(\omega)$, where the constant $C_{0}>0$ may depend on the specific market and the arbitrageur. It is then clear that there is an arbitrage opportunity at date $t$ after discounting the transaction costs if and only if

$$
P\left(\left\{\omega \in \Omega \mid l^{t}(\omega)>C_{0}\right\}\right)>0 .
$$

We now follow the approach of Jouini and Kallal (1995) incorporating bidask spreads. As them, we assume that there are two different $\mathbb{R}^{n}$-valued adapted stochastic processes $v^{t}$ and $c^{t}$ such that the inequalities $0 \leq v_{i}^{t}(\omega) \leq$ $c_{i}^{t}(\omega)$ hold a.e. for every $t$. For a given portfolio $x$ at date $t$, the price of $x$ is determined by $\Pi^{t}(x, \omega)=\sum_{i=1}^{n} x_{i}^{t}(\omega) q_{i}^{t}(\omega)$, where $q_{i}^{t}(\omega)=c_{i}^{t}(\omega)$ whenever $x_{i}(\omega) \geq 0$ and $q_{i}^{t}(\omega)=v_{i}^{t}(\omega)$ otherwise. So, the function $\Pi$ is sublinear and convex. Definition 2.1 needs some minor modifications. 
Definition 6.1. The portfolio $x \in\left(L^{2}\left(\Sigma_{t}\right)\right)^{n}$ is said to be an arbitrage opportunity (second type) at date $t$ if there exists $A \in \Sigma_{t}$ with $P(A)>0$ such that $\Pi^{t}(x, \omega)<0$ a.e. in $A$ and $\Pi^{t+1}(-x, \omega) \leq 0$ a.e. in $A$.

It is worthwhile to mention that comments before Definition 2.2 also apply here, and consequently, the definition above may be understood as a definition of dynamic arbitrage, not necessarily equivalent to the concept of free lunch.

The main results of Sections 2 and 3 can be easily generalized and thus we only summarize the main modifications. The functions $g$ and $f$ become respectively

$$
g_{b a}(x, \omega)=\frac{-\Pi^{t}(x, \omega)}{\Pi^{t}\left(x^{+}, \omega\right)-\Pi^{t}\left(-x^{-}, \omega\right)} \text { and } f_{b a}(x, \omega)=\frac{\Pi^{t}(x, \omega)}{\Pi^{t}\left(-x^{-}, \omega\right)}
$$

with the convention that $f=0$ or $g=0$ if denominators vanish. The measure $l_{b a}^{t}$ is now defined as the strong solution of the readapted problem

$$
\max g_{b a}(x, \omega) \text { s.t } \Pi^{t+1}(-x, \omega) \leq 0
$$

while $m_{b a}^{t}$ is the strong solution of

$$
\max f_{b a}(x, \omega) \mathrm{s} . \mathrm{t} \Pi^{t+1}(-x, \omega) \leq 0 .
$$

The linear problems under short-selling restrictions leading to the measures $m^{t}$ and $\mathfrak{M}^{t}$ become respectively the following concave problems

$$
\begin{aligned}
& \max -\Pi^{t}(x, \omega) \\
& \qquad \begin{array}{l}
\text { s.t. } \Pi^{t+1}(-x, \omega) \leq 0 \\
\\
\quad \sum_{i=1}^{n} h_{i} v_{i}^{t}=1 \\
x_{i} \geq-h_{i} \quad i=1, \ldots, n \\
x_{i} \in L^{2}\left(\Sigma^{t}\right), \quad h_{i} \in L_{+}^{2}\left(\Sigma^{t}\right) \quad i=1, \ldots, n
\end{array}
\end{aligned}
$$


and

$$
\begin{aligned}
\max - & \int_{\Omega} \Pi^{t}(x, \omega) d P \\
\text { s.t. } \Pi^{t+1}(-x, \omega) \leq 0 & \\
& \sum_{i=1}^{n} h_{i} v_{i}^{t}=1 \\
& x_{i} \geq-h_{i} \quad i=1, \ldots, n \\
& x_{i} \in L^{2}\left(\Sigma^{t}\right), \quad h_{i} \in L_{+}^{2}\left(\Sigma^{t}\right) \quad i=1, \ldots, n
\end{aligned}
$$

and yield the measures $m_{b a}^{t}$ and $\mathfrak{M}_{b a}^{t}$. As in the frictionless case, it can be proved that, if the portfolio $x^{*}$ solves the problem leading to $\mathfrak{M}_{b a}^{t}$, then $x^{*}$ also solves all the problems leading to $m_{b a}^{t}$ and $l_{b a}^{t}$. Furthermore, a duality theory can be also stated under bid-ask spread assumptions. Since latter problems are concave, applying the theory of duality in convex programming, see for instance Khanh (1995), Balbás and Guerra (1996) or Kuk et al. (1996), we obtain similar results to Theorem 4.2 for the corresponding problems

$$
\begin{aligned}
& \min \alpha(\omega) \\
& \text { s.t. } v_{i}^{t} \leq E\left(f c_{i}^{t+1} \mid \Sigma^{t}\right)+l_{i} \quad i=1, \ldots, n \\
& \quad E\left(f v_{i}^{t+1} \mid \Sigma^{t}\right)+l_{i} \leq c_{i}^{t} \quad i=1, \ldots, n \\
& \quad \alpha v_{i}^{t} \geq l_{i} \quad i=1, \ldots, n \\
& \alpha \in L_{+}^{2}\left(\Sigma^{t}\right), \quad l_{i} \in L_{+}^{2}\left(\Sigma^{t}\right) \quad f \in L_{+}^{2}\left(\Sigma^{t+1}\right) \quad i=1, \ldots, n
\end{aligned}
$$

and

$$
\begin{aligned}
& \min \int_{\Omega} \alpha(\omega) d P \\
& \text { s.t. } v_{i}^{t} \leq E\left(f c_{i}^{t+1} \mid \Sigma^{t}\right)+l_{i} \quad i=1, \ldots, n \\
& E\left(f v_{i}^{t+1} \mid \Sigma^{t}\right)+l_{i} \leq c_{i}^{t} \quad i=1, \ldots, n \\
& \alpha v_{i}^{t} \geq l_{i} \quad i=1, \ldots, n \\
& \alpha \in L_{+}^{2}\left(\Sigma^{t}\right), \quad l_{i} \in L_{+}^{2}\left(\Sigma^{t}\right) \quad f \in L_{+}^{2}\left(\Sigma^{t+1}\right) \quad i=1, \ldots, n
\end{aligned}
$$

Dual problems provide an interpretation in terms of state prices as in the frictionless case and also the main procedure to compute the measures in practice since both problems are linear. 
Finally we can introduce a third assumption on the frictions combining linear taxes on the total value of trade (e.g., fees paid to brokers) and bidask spreads. It is easily deduced a measure $l_{f r}^{t}$ of the level of arbitrage opportunities as

$$
l_{f r}^{t}(\omega)=\max \left\{0, l_{b a}^{t}(\omega)-C_{0}\right\}
$$

\section{Measuring the arbitrage of the first type}

The measures introduced above are useful to analyze arbitrage portfolios of the second type but there might be situations where the measures vanish in presence of arbitrage of the first type. To solve this difficulty one only has to consider an optimization problem such that the objective function incorporates the arbitrage earnings attainable at date $t+1$. There are several possibilities and, so for instance, we can maximize the expected value and the conditional expectation of

$$
\sum_{i=1}^{n} x_{i} p_{i}^{t+1}
$$

among the arbitrage portfolios providing at $t$ the random relative profit $m^{t}(\omega)$. Concretely, we consider the vector optimization problem

$$
\begin{array}{rl}
\max \sum_{i=1}^{n} & E\left(x_{i} p_{i}^{t+1} \mid \Sigma_{t}\right) \\
\text { s.t. } & \sum_{i=1}^{n} x_{i} p_{i}^{t}=-m^{t} \quad \text { a.e. } \\
& \sum_{i=1}^{n} x_{i} p_{i}^{t+1} \geq 0 \quad \text { a.e. } \\
& \sum_{i=1}^{n} h_{i} p_{i}^{t}=1 \quad \text { a.e. } \\
& x_{i} \geq-h_{i} \quad \text { a.e., } i=1,2, \ldots, n \\
& x_{i} \in L^{2}\left(\Sigma_{t}\right), h_{i} \in L_{+}^{2}\left(\Sigma_{t}\right) \quad i=1,2, \ldots, n
\end{array}
$$


and the scalar problem

$$
\begin{aligned}
\max & \sum_{i=1}^{n} \int_{\Omega} x_{i} p_{i}^{t+1} d P \\
\text { s.t. } & \sum_{i=1}^{n} x_{i} p_{i}^{t}=-m^{t} \quad \text { a.e. } \\
& \sum_{i=1}^{n} x_{i} p_{i}^{t+1} \geq 0 \quad \text { a.e. } \\
& \sum_{i=1}^{n} h_{i} p_{i}^{t}=1 \quad \text { a.e. } \\
& x_{i} \geq-h_{i} \quad \text { a.e., } \quad i=1,2, \ldots, n \\
& x_{i} \in L^{2}\left(\Sigma_{t}\right), h_{i} \in L_{+}^{2}\left(\Sigma_{t}\right) \quad i=1,2, \ldots, n
\end{aligned}
$$

It may be easily proved that the solution for both problems is attained at the same portfolio, and it is also important to point out that this solution is a strong one for the vector problem and also solves problem $(I V)$. Denoting by $k^{t}$ and $K^{t}$ the optimal values respectively, we consider the stochastic and numerical measures $m^{t}+k^{t}$ and $\mathfrak{M}^{t}+K^{t}$. It trivially follows that both measures vanish if and only if there are no arbitrage opportunities (of any kind) between $t$ and $t+1$.

\section{Conclusions}

The paper has developed three new measures, $\mathfrak{M}, m$ and $l$, in order to quantify in monetary terms the lack of the absence arbitrage in a financial market. They vanish if and only if the arbitrage absence holds and they increase from 0 to 1 as the relative arbitrage profit grows. The theory is built in a very general setting and applies for static and discrete time dynamic asset pricing models. Thus, all the measures become stochastic.

Many empirical papers analyze the existence of arbitrage by testing concrete well known strategies (violations of the put-call parity for European options, the relationship between spot and futures prices etc.). The measures introduced here provide a more general methodology. In fact, by computing the measures one is incorporating all the available assets and all the possible arbitrage portfolios are being tested. 
Many sort of frictions may be discounted and, thus, the measures seem to be very adequate to analyze the imperfect market case. In particular the theory also applies when we assume that market frictions depend on the total volume of trade, or when we consider the case proposed by Jouini and Kallal (1995) which, as pointed out by these authors, contains many different possibilities on market frictions. Furthermore, our measures also permit us to incorporate combinations of both type of frictions.

The measures are introduced by means of several dual pairs of vector optimization problems. Thus, from a practical viewpoint, concrete (an optimal) arbitrage portfolios are obtained (if they exist) when testing the measures. This may be very useful to traders and researchers when empirically testing real markets.

From a theoretical viewpoint, primal problems allow us to interpret the measures in terms of arbitrage gains and provide necessary and sufficient conditions to guarantee the arbitrage absence in the imperfect case. If we assume the absence of duality gaps then dual problems also characterize the arbitrage absence in the imperfect case, permit us to interpret the measures in terms of "frictions effect" or "committed errors" in the valuation process and provide pricing rules and risk-neutral probabilities (or a proxy for them) even for no-arbitrage free imperfect markets.

Some new properties appeared in the paper may be also interesting in Vector Optimization Theory since a procedure to solve a special kind of non-differentiable problem is provided, and results on its sensitivity and the absence of duality gap are obtained too.

\section{References}

Anderson E.J. and Nash P. (1987). Linear Programming in Infinite-Dimensional Spaces. John Wiley.

Balbás A. and Guerra P.J. (1996). Sensitivity Analysis for Convex Multiobjective Programming in Abstract Spaces. Journal of Mathematical Analysis and Applications 202, 645-658.

Balbás A., Guerra P.J. and Muñoz-Bouzo M.J. (2002a). The Balance Space Approach in Optimization with Riesz Spaces Valued Objectives. An Application to Financial Markets. Computers and Mathematics with Applications 44, 887897. 
Balbás A., Longarela I.R. and Lucia J. (1999). How Financial Theory Applies to Catastrophe-Linked Derivatives: An Empirical Test of Several Pricing Models. Journal of Risk and Insurance 66, 551-582.

Balbás A., Longarela I.R. and Pardo A. (2000). Integration and Arbitrage in the Spanish Financial Markets: An Empirical Approach. Journal of Futures Markets 20, 321-344.

Balbás A., Mirás M. and Muñoz-Bouzo M.J. (2002b). Projective System Approach to the Martingale Characterization of the Absence of Arbitrage. Journal of Mathematical Economics 37, 311-323.

Balbás A. and Muñoz-Bouzo M.J. (1998). Measuring the Degree of Fulfillment of the Law of One Price. Applications to Financial Markets Integration. Investigaciones Económicas 22, 19-44.

Chateauneuf A., Kast R. and Lapied A. (1996). Choquet Pricing for Financial Markets with Frictions. Mathematical Finance 6, 323-330.

Chen Z. and Knez P.J. (1995). Measurement of Market Integration and Arbitrage. The Review of Financial Studies 8, 287-325.

Clark S.A. (1993). The Valuation Problem in Arbitrage Price Theory. Journal of Mathematical Economics 22, 463-478.

Davis M.H.A., Panas V.G. and Zariphopoulou T. (1993). European Option Pricing with Transaction Costs. SIAM Journal of Control and Optimization 31, 470493.

De Wagenaere A. and Wakker P.P. (2001). Nonmonotonic Choquet Integrals. Journal of Mathematical Economics 36, 45-60.

Harrison J. and Kreps D. (1979). Martingales and Arbitrage in Multiperiod Securities Markets. Journal of Economic Theory 20, 381-408.

Ingersoll J.E., Jr (1987). Theory of Financial Decision Making. Rowman and Littlefield Publishers, Inc.

Jaschke S.R. (1998). Arbitrage Bounds for the Term Structure of Interest Rates. Finance and Stochastics 2, 29-40.

Jouini E. and Kallal H. (1995). Martingales and Arbitrage in Securities Markets with Transaction Costs. Journal of Economic Theory 66, 178-197.

Kamara A. and Miller T.W., Jr (1995). Daily and Intradaily Tests of European Put-Call Parity. Journal of Financial and Quantitative Analysis 30, 519-541.

Kempf A. and Korn O. (1998). Trading System and Market Integration. Journal of Financial Intermediation 7, 220-239.

Khanh P.O. (1995). Sufficient Optimality Conditions and Duality in Vector Op- 
timization With Invex-Convexlike Functions. Journal of Optimization Theory and Applications 87, 359-368.

Kuk H., Tanino J. and Tanaka M. (1996). Sensitivity Analysis in Vector Optimization. Journal of Optimization Theory and Applications 89, 713-730.

Lee J.H. and Nayar N. (1993). A Transactions Data Analysis of Arbitrage between Index Options and Index Futures. The Journal of Futures Markets 13, 889902 .

Luenberger D.G. (1969). Optimization by Vector Spaces Methods. John Wiley.

Moulin H. (1979). Fondation de la Théorie de Jeux. Hermann.

Pardo A., Balbás A. and Meneu V. (2002). The Effectiveness of Several Market Integration Measures When Facing a Market Turmoil. Derivatives Use, Trading and Regulation (to appear).

Pham H. and Touzi N. (1999). The Fundamental Theorem of Asset Pricing with Cone Constraints. Journal of Mathematical Economics 31, 265-279.

Prisman E.Z. (1986). Valuation of Risky Assets in Arbitrage Free Economies with Frictions. The Journal of Finance, 41, 545-56.

Protopapadakis A. and Stoll H.R. (1983). Spot and Future Prices and the Law of One Price. The Journal of Finance, 38, 1431-1455.

Sawaragi Y., Nakayama H. and Tanino T. (1985). Theory of Multiobjective Optimization. Academic Press.

Toft K.B. (1996). On the Mean-Variance Trade-off in Option Replication with Transaction Costs. Journal of Financial and Quantitative Analysis 31, 233263.

Zowe S. (1975). A Duality Theorem for a Convex Programming Problem in Order Complete Vector Lattices. Journal of Mathematical Analysis and Applications $50,283-287$. 